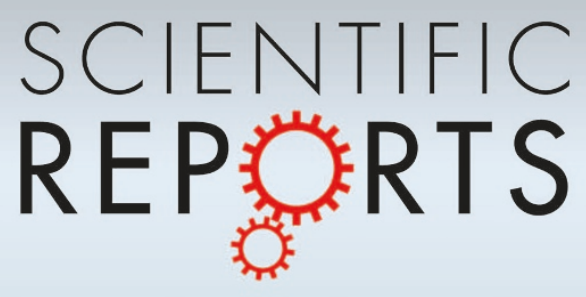

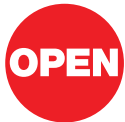

SUBJECT AREAS: PHOTOCATALYSIS

NANOPARTICLES

ELECTRONIC PROPERTIES AND MATERIALS

STRUCTURAL PROPERTIES

Received

27 June 2012

Accepted

4 February 2013

Published

26 March 2013

Correspondence and requests for materials should be addressed to

X.C. (chenxiaobo@

umkc.edu) or S.S.M. (ssmao@me.berkeley.

edu)

\title{
Properties of Disorder-Engineered Black Titanium Dioxide Nanoparticles through Hydrogenation
}

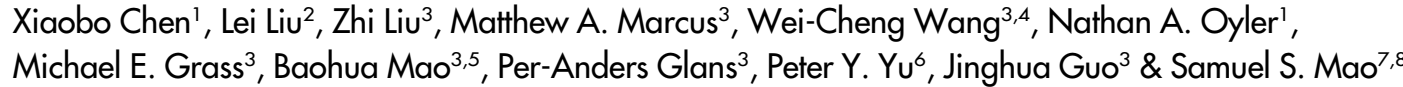

'University of Missouri - Kansas City, Department of Chemistry, Kansas City, MO 641 10, USA, ${ }^{2}$ State Key Laboratory of Luminescence and Applications, Changchun Institute of Optics, Fine Mechanics and Physics, Chinese Academy of Sciences, 3888 Dongnanhu Road, Changchun, Jilin 130033, China, ${ }^{3}$ Advanced Light Source, Lawrence Berkeley National Laboratory, Berkeley, CA 94720, USA, ${ }^{4}$ Department of Physics, Tamkang University, Tamsui, Taiwan 250, China, ${ }^{5}$ Institute of Functional Nano \& Soft Materials Laboratory, Soochow University, Suzhou, Jiangsu 215123, China, ${ }^{6}$ Department of Physics, University of California at Berkeley, Berkeley, CA 94720, USA, 'Department of Mechanical Engineering, University of California at Berkeley, Berkeley, CA 94720, USA, ${ }^{8}$ Environmental Energy Technology Division, Lawrence Berkeley National Laboratory, Berkeley, CA 94720, USA.

The recent discovery of "black" $\mathrm{TiO}_{2}$ nanoparticles with visible and infrared absorption has triggered an explosion of interest in the application of $\mathrm{TiO}_{2}$ in a diverse set of solar energy systems; however, what a black $\mathrm{TiO}_{2}$ nanoparticle really is remains a mystery. Here we elucidate more properties and try to understand the inner workings of black $\mathrm{TiO}_{2}$ nanoparticles with hydrogenated disorders in a surface layer surrounding a crystalline core. Contrary to traditional findings, $\mathrm{Ti}^{3+}$ here is not responsible for the visible and infrared absorption of black $\mathrm{TiO}_{2}$, while there is evidence of mid-gap states above the valence band maximum due to the hydrogenated, engineered disorders. The hydrogen atoms, on the other hand, can undergo fast diffusion and exchange. The enhanced hydrogen mobility may be explained by the presence of the hydrogenated, disordered surface layer. This unique structure thus may give $\mathrm{TiO}_{2}$, one of the most-studied oxide materials, a renewed potential.

- $\mathrm{iO}_{2}$ has attracted substantial interest as a photocatalyst due to its activity for breaking water into oxygen and hydrogen under ultraviolet (UV) light irradiation ${ }^{1,2}$. However, its overall efficiency for solar-driven photocatalysis is very limited, because of its wide bandgap in the UV, which accounts for less than $5 \%$ of the total solar irradiation ${ }^{3,4}$. Much effort has been made to improve its optical absorption for its photocatalytic activity, with metal, non-metal, or self-doping ${ }^{3-9}$. The recent realization of black $\mathrm{TiO}_{2}$ nanoparticles ${ }^{10}$ has opened a new avenue to the long-wavelength optical absorption and triggered a great amount of interest ${ }^{11-14}$. However, our understanding of this new black $\mathrm{TiO}_{2}$ remains far from satisfactory. In this study, we attempt to improve our understanding of this black $\mathrm{TiO}_{2}$ nanoparticle by using comprehensive advanced characterization techniques together with ab-initio calculations. The results yield exciting insights on the properties of black $\mathrm{TiO}_{2}$ that may be applicable to other oxide nanomaterials.

It is well known that $\mathrm{Ti}^{3+}$ impurities and oxygen vacancy defects turn white $\mathrm{TiO}_{2}$ yellow or blue ${ }^{3-9,15}$, and it would be natural and reasonable to think that probably $\mathrm{Ti}^{3+}$ is also responsible for the black $\mathrm{TiO}_{2}$, as this material is obtained by reacting white $\mathrm{TiO}_{2}$ with hydrogen and in the bulk anatase phase, the presence of atomic hydrogen was found to yield $\mathrm{Ti}^{3+}$ centers ${ }^{21}$. Hydrogen adsorption was proposed to proceed through molecular $\mathrm{H}_{2}$ dissociation via a hydride intermediate and subsequent migration, and studies of hydrogen adsorption on $\left(\mathrm{TiO}_{2}\right)_{\mathrm{N}}$ clusters revealed that an initial hydrogen atom could easily bond to a terminal oxygen $\operatorname{site}^{13}$. The observed hydrogen diffusion into the $\mathrm{TiO}_{2}(110)$ surface with a barrier of approximately $1 \mathrm{eV}$ suggests that hydrogen incorporation into the $\mathrm{TiO}_{2}$ lattice could take place at room temperature ${ }^{16-20}$. Depending on whether the crystal surface terminations are predominantly (001) or (101), hydrogen can penetrate the surface and be incorporated into the lattice easily ${ }^{16,22}$.

However, the black $\mathrm{TiO}_{2}$ here is actually different, in that no detectable $\mathrm{Ti}^{3+}$ was found based on our previous laboratory-source X-ray photoelectron spectroscopy (XPS) results ${ }^{10}$, and more experimental evidence shown herein. Furthermore strong evidence testifies that the additional valence band edge states in the black $\mathrm{TiO}_{2}$ which are believed to be responsible for the long-wavelength absorption of the black $\mathrm{TiO}_{2}$ go away when $\mathrm{Ti}^{3+}$ is induced by extended-period X-ray irradiation. In addition, we reveal important properties of the hydrogen and the 

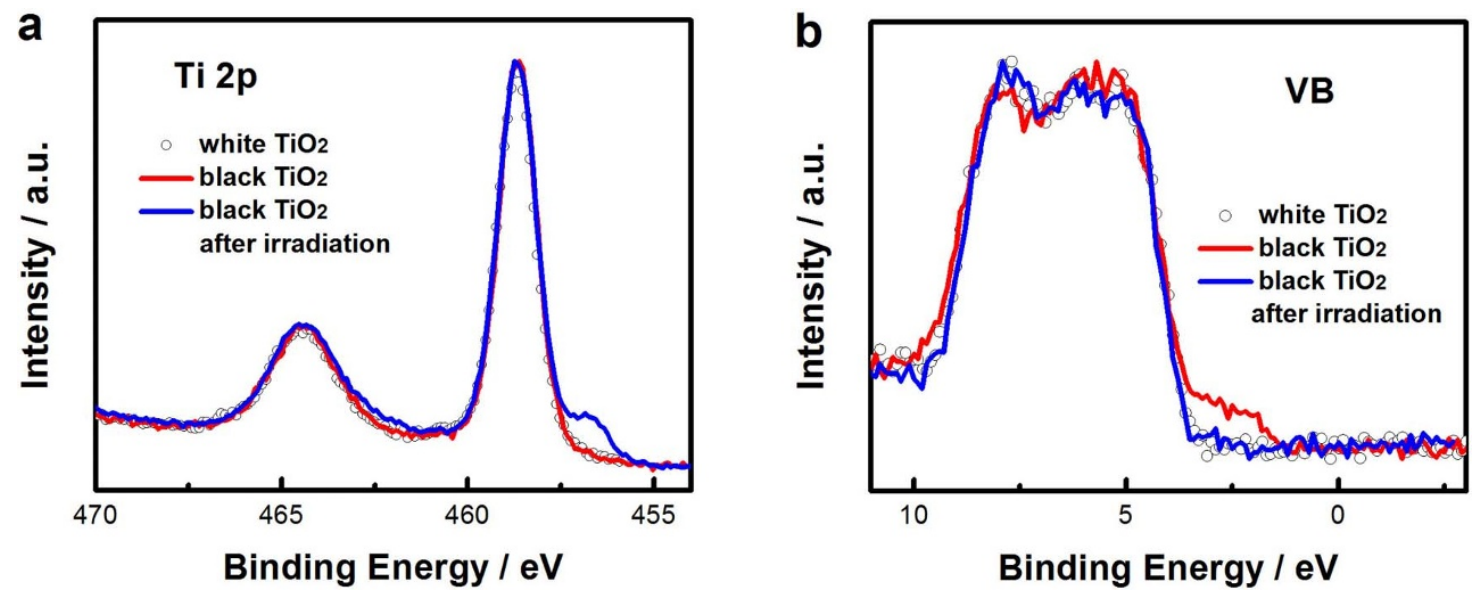

Figure 1 Synchrotron XPS spectra of white and black $\mathrm{TiO}_{2}$. (a) $\mathrm{Ti} 2 \mathrm{p}$ XPS spectra of white and hydrogenated black $\mathrm{TiO}_{2}$, before and after three hours of $635 \mathrm{eV}$ X-ray illumination. (b) Valence band (VB) XPS spectra of white and hydrogenated black $\mathrm{TiO}_{2}$, before and after three hours of $635 \mathrm{eV}$ X-ray illumination.

disorder in the black $\mathrm{TiO}_{2}$, although a definitive conclusion is still under investigation. We believe that our study would shine more lights on this new material or the alike.

\section{Results}

From high signal-to-noise ratio synchrotron X-ray spectroscopy, we found that both the post-hydrogenated black and pristine white $\mathrm{TiO}_{2}$
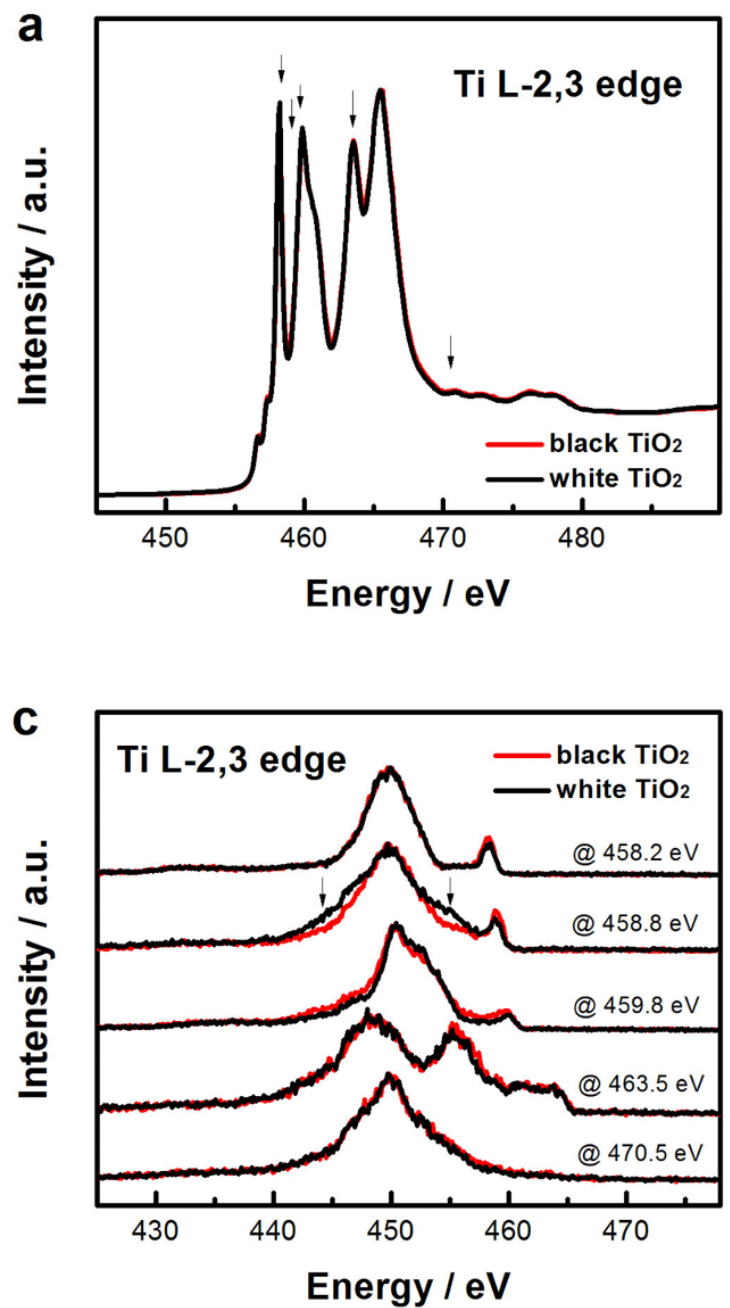

nanoparticles are free of $\mathrm{Ti}^{3+}$ species, as evident from the almost identical Ti $2 p$ XPS spectra taken at $635 \mathrm{eV}$ and the absence of $\mathrm{Ti}^{3+}$ feature (Fig. 1). To examine the possible presence of impurities in the hydrogenated black $\mathrm{TiO}_{2}$, we performed XPS measurements as well as XAS at the $\mathrm{C}$ and $\mathrm{N}$ K-edges (Supplementary, Fig. S1). Both XPS and XAS spectra show similar spectral profile for the white and black $\mathrm{TiO}_{2}$, which essentially eliminates the possibility of an impurity contribution.
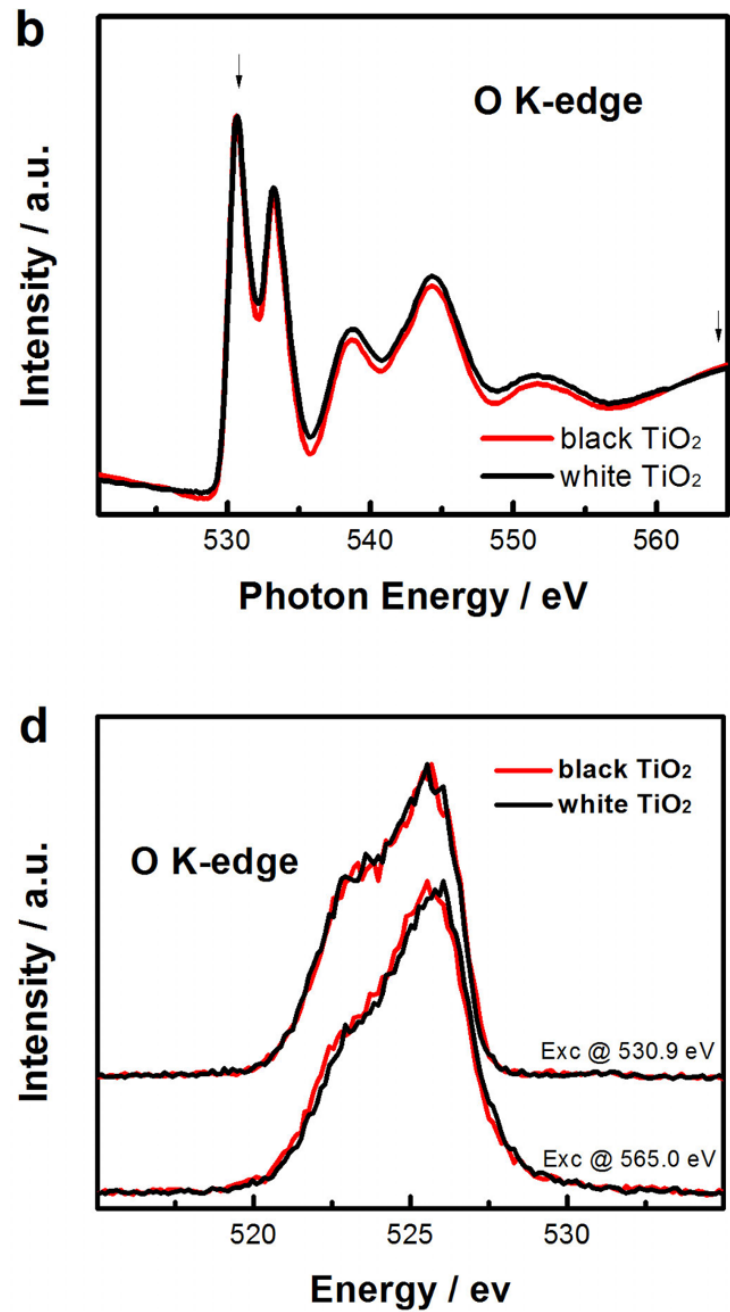

Figure $2 \mid$ Synchrotron spectra of white and black $\mathrm{TiO}_{2}$. (a) Ti L-edge XAS, (b) O K-edge XAS, (c) Ti L-edge XES, (d) O K-edge XES. (The arrows in (a) and (b) show the excitation energies for (c) and (d), respectively.) 
On the other hand, we found that the appearance of $\mathrm{Ti}^{3+}$ actually is accompanied with the disappearance of the additional valence bandedge states which are believed to be responsible for the long-wavelength absorption of the black $\mathrm{TiO}_{2}$, and thus the $\mathrm{Ti}^{3+}$ does not contribute to the additional valence band-edge states on the black $\mathrm{TiO}_{2}$. Fig. $1 \mathrm{~b}$ shows that the hydrogenated black $\mathrm{TiO}_{2}$ exhibits additional electronic states above the valence band maximum of the white $\mathrm{TiO}_{2}$ nanocrystals ${ }^{10}$. These additional electronic states are believed to be responsible for the long-wavelength absorption of the black $\mathrm{TiO}_{2}$. Changes of XPS spectrum are observed upon an extended period (3 hours) of X-ray irradiation on the black $\mathrm{TiO}_{2}$. A small peak emerges at the high energy side of the Ti $2 p$ XPS peak at $457.0 \mathrm{eV}$ (Fig. 1a), while at the same time, the states above the valence-band maximum and characteristic of the black $\mathrm{TiO}_{2}$ disappear and the valence band XPS of the black and white $\mathrm{TiO}_{2}$ nanoparticles become identical (Fig. 1b). The Ti $2 p$ XPS peak at $457.0 \mathrm{eV}$ is characteristic of $\mathrm{Ti}^{3+}$ species. These findings provides the direct evidence that $\mathrm{Ti}^{3+}$ states are not responsible for mid-gap electronic states above the valence band of the hydrogenated black $\mathrm{TiO}_{2}$.

To further confirm the absence of $\mathrm{Ti}^{3+}$ in the black $\mathrm{TiO}_{2}$, we conducted other measurements in overcoming the limitation of the XPS technique, which normally only yields information about $1 \mathrm{~nm}$ deep. Soft X-ray XAS and X-ray emission spectroscopy (XES) have probe depths of tens of nanometers. Figs. $2 \mathrm{a}$ and $2 \mathrm{~b}$ show the Ti $L$ edge and $\mathrm{O} K$-edge XAS spectra of the black and white $\mathrm{TiO}_{2}$. The Ti
$L$-edge XAS consists of contributions from $L_{3}\left(2 p_{3 / 2}-3 d\right)$ (457$462 \mathrm{eV})$ and $L_{2}\left(2 p_{1 / 2}-3 d\right)(462-467 \mathrm{eV})$ transitions ${ }^{23}$. The Ti L-edge absorption spectra again indicate no obvious sign of $\mathrm{Ti}^{3+}$ and almost identical absorption features in both black and white $\mathrm{TiO}_{2}$. The oxygen $K$-edge XAS spectra can be divided into a well-defined preedge region attributed to $\mathrm{O} 2 p$ states with $\mathrm{Ti} 3 d$ - $\mathrm{O} 2 p$ mixing, and the region above $536 \mathrm{eV}$, representing $\mathrm{O} 2 p$ states hybridized with $\mathrm{Ti} 4 s$ and $4 p$ states. The similar $\mathrm{O} K$-edge absorption spectra indicate that hybridization between $\mathrm{Ti} 3 d$ and $\mathrm{O} 2 p$ states in both the black and white $\mathrm{TiO}_{2}$ is extensive.

Figs. $2 \mathrm{c}$ and $2 \mathrm{~d}$ shows the Ti $L$-edge and $\mathrm{O} K$-edge XES spectra of the black and white $\mathrm{TiO}_{2}$. The Ti $L$-edge XES is attributed to the filling of the $\mathrm{Ti} 2 p$ core hole from the $\mathrm{Ti} 3 d$, while the $\mathrm{O} K$-edge emission comes from filling of an $\mathrm{O} 1 s$ hole from the $\mathrm{O} 2 p$ orbitals. Both relaxations are affected by the couplings between the Ti $3 d$ and O $2 p$ orbitals. Both black and white $\mathrm{TiO}_{2}$ show similar emission patterns excited at various energies, which suggests that the nature of the couplings in both black and white $\mathrm{TiO}_{2}$ is similar. The weaker peak in the $\mathrm{Ti} L$-edge XES spectrum at the resonant excitation energy of $458.8 \mathrm{eV}$ of the black $\mathrm{TiO}_{2}$ may indicate that the coupling between the $\mathrm{Ti} 3 d$ and $\mathrm{O} 2 p$ orbitals of the black $\mathrm{TiO}_{2}$ is slightly weaker due to the shared-bonding with the hydrogen and oxygen.

At this moment, by combining the information based on the almost identical Ti and O core-level XPS, soft X-ray XAS and XES results of black and white $\mathrm{TiO}_{2}$, we can conclude that the $\mathrm{Ti}^{3+}$ is not
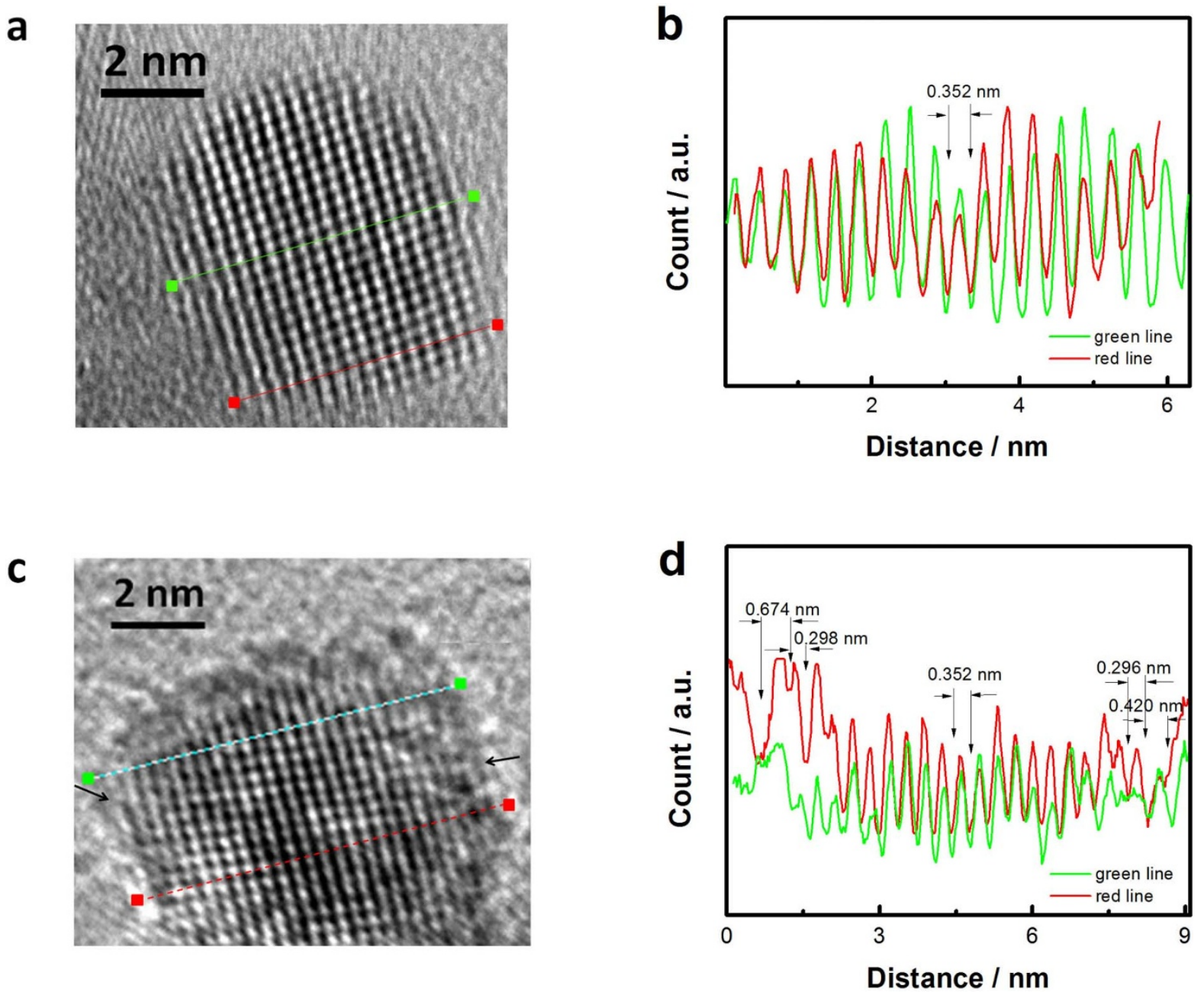

Figure 3 High-resolution transmission electron microscope (HRTEM) images and structural analysis of white and black TiO ${ }_{2}$ nanocrystals. HRTEM (a) and line analyses (b) of white $\mathrm{TiO}_{2}$. HRTEM (c) and line analyses (d) of black $\mathrm{TiO}_{2}$. The zeros of the axis in (b) and (d) correspond to the left ends of the lines in (a) and (c). The red, and green curves in (b) and (d) correspond to the red and green lines in (a) and (c). 
detected in black $\mathrm{TiO}_{2}$ within the technique accuracy and information depth employed in this study. This finding is also supported by the almost identical Ti K-edge X-ray near edge absorption spectra (XANES) of the black and white $\mathrm{TiO}_{2}$ (Supplementary, Fig. S2).

\section{Discussion}

In order to get spatially-resolved information on the structural changes in the black $\mathrm{TiO}_{2}$, we performed high-resolution transmission electron microscope (HRTEM) studies of the black and white $\mathrm{TiO}_{2}$ nanoparticles. The white $\mathrm{TiO}_{2}$ is completely crystalline, displaying clearly-resolved and well-defined lattice fringes, even at the surface of the nanocrystal (Fig. 3a). The distance between the adjacent lattice planes is $0.352 \mathrm{~nm}$, typical for anatase, uniform throughout the whole nanocrystal (Fig. 3b, and supplementary, Fig. S3). The black $\mathrm{TiO}_{2}$ nanocrystal has a crystalline-disordered core-shell structure (Fig. 3c). Structural deviation from the standard crystalline anatase at the outer layer is readily seen, where the straight lattice line is bent at the edge of the nanoparticle. While the core shows wellresolved (101) lattice plane with typical anatase plane distance, on the disordered outer layer, the distances between adjacent lattice planes are no longer uniform (Fig. 3d, and supplementary, Fig. S4). This structural difference would verify the disordered nature of the surface layer of the black $\mathrm{TiO}_{2}$. We prepared the grids for both samples under identical conditions, and the samples were relatively stable under the beam during the measurements, which were done under the same conditions. Therefore, we believe that the observed differences between black and white $\mathrm{TiO}_{2}$ are characteristic of the materials, rather than artifacts of sample preparation or microscopy.
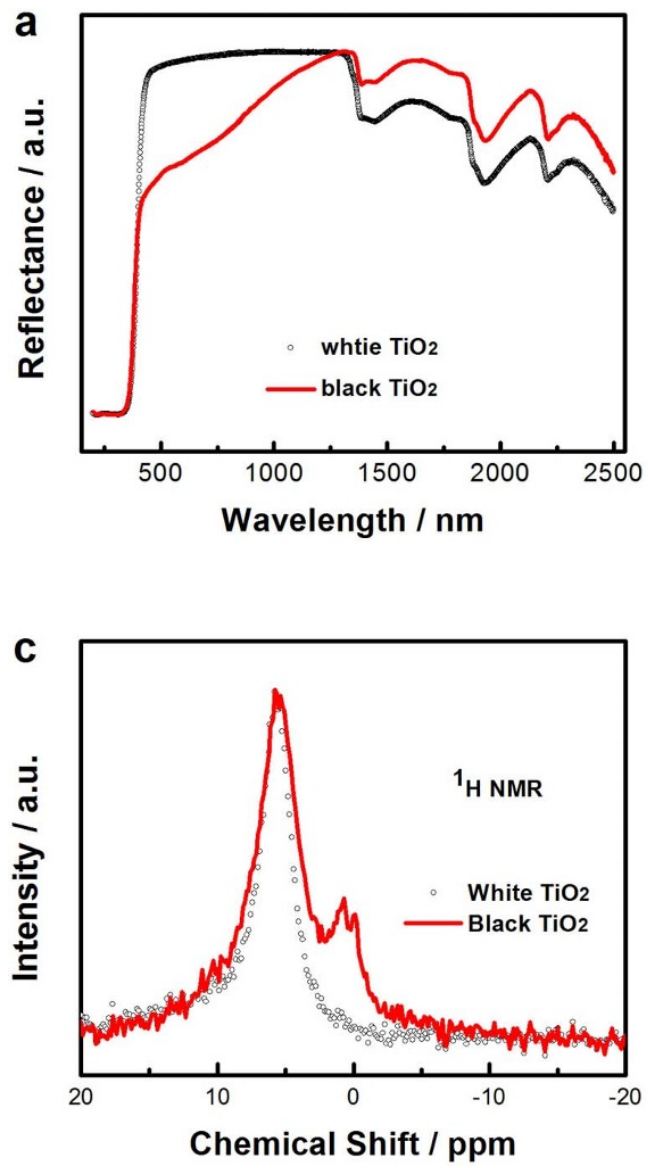

In order to understand the role of hydrogen in the black $\mathrm{TiO}_{2}$, as the black $\mathrm{TiO}_{2}$ is obtained from reaction of white $\mathrm{TiO}_{2}$ with hydrogen, we first measured wide-range optical spectra from UV (200 nm) to the far-infrared $\left(700 \mathrm{~cm}^{-1}\right)$ of both black and white $\mathrm{TiO}_{2}$. The main difference between the optical spectra is in the visible and nearinfrared regions from 400 to $1100 \mathrm{~nm}$, where black $\mathrm{TiO}_{2}$ is optically absorbing (Fig. 4a). Both materials show similar absorption features from $1100 \mathrm{~nm}$ to $2500 \mathrm{~nm}$ (or $4000 \mathrm{~cm}^{-1}$ ), and both exhibit $\mathrm{OH}$ absorption bands near the $3400 \mathrm{~cm}^{-1}$ region, due to a bridging $\mathrm{OH}$ group (Fig. 4b). The peaks at around $3700 \mathrm{~cm}^{-1}$ are due to the $\mathrm{O}-\mathrm{H}$ stretching and wagging modes. The strength of the terminal $\mathrm{O}-\mathrm{H}$ mode is reduced after white $\mathrm{TiO}_{2}$ is converted to black $\mathrm{TiO}_{2}$, which suggests that the hydrogen incorporated into the $\mathrm{TiO}_{2}$ possibly does not passivate a significant number of $\mathrm{O}$ dangling bonds as this would otherwise increases the absorption. The wider $\mathrm{OH}$ absorption band of the black $\mathrm{TiO}_{2}$ indicates that the $\mathrm{OH}$ groups experience a more varied environment on the disordered black $\mathrm{TiO}_{2}$ surface than on the crystalline surface of the white $\mathrm{TiO}_{2}$. The linewidth difference may, in addition to the inhomogeneous effect referred to above, come in part from homogeneous (lifetime) broadening due to rapid exchange among sites.

We further conducted ${ }^{1} \mathrm{H}$ NMR measurements to examine the role of hydrogen in black $\mathrm{TiO}_{2}$ (Figs. 4c). Both the black and white $\mathrm{TiO}_{2}$ show a large peak at chemical shift of $+5.7 \mathrm{ppm}$. The slightly larger linewidth in black $\mathrm{TiO}_{2}$ may be caused by the incorporation of $\mathrm{H}$ at bridging sites at the disordered phase produced during the hydrogenation process, or may be due to the bridging sites located on different crystallographic planes on the surface ${ }^{24}$. Compared to white $\mathrm{TiO}_{2}$, black $\mathrm{TiO}_{2}$ shows two additional narrow peaks at chemical

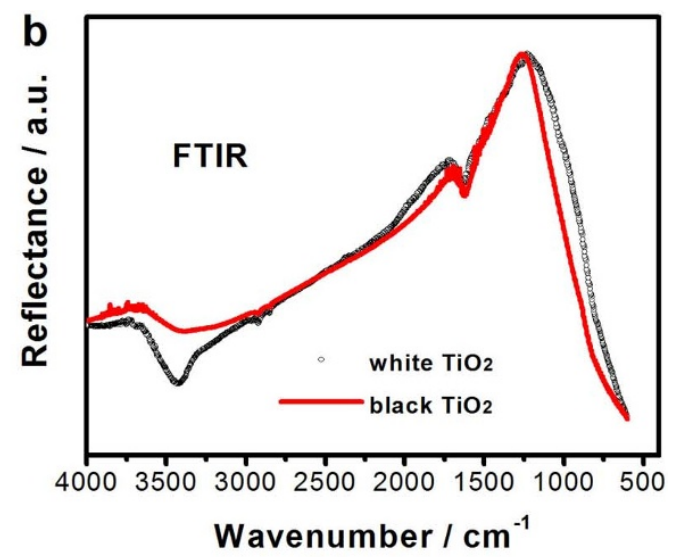

Figure 4 Optical and vibrational spectra of white and black $\mathrm{TiO}_{2}$. (a) Optical reflectance spectra of hydrogenated black $\mathrm{TiO}_{2}$ nanocrystals compared with white $\mathrm{TiO}_{2}$ nanocrystals. (b) FTIR reflectance spectra of hydrogenated black $\mathrm{TiO}_{2}$ nanocrystals compared with white $\mathrm{TiO}_{2}$ nanocrystals. (c) ${ }^{1} \mathrm{H}$ magic angle NMR spectra of hydrogenated black $\mathrm{TiO}_{2}$ nanocrystals compared with white $\mathrm{TiO}_{2}$ nanocrystals. 
shifts of $0.73 \mathrm{ppm}$ and $-0.03 \mathrm{ppm}$. These small and sharp ${ }^{1} \mathrm{H}$ NMR resonances suggests that the hydrogen concentration is low and, there are dynamical exchanges between hydrogen in the different environments in black $\mathrm{TiO}_{2}$. Some examples of such dynamical exchange mechanisms are rapid isotropic diffusion and rapid exchange between similar proton environments ${ }^{25,26}$. These two peaks are probably associated with hydrogen located in the hydrogenated, disordered surface layer of the black $\mathrm{TiO}_{2}$ as a result of hydrogenation. Thus the presence of disordered surface layer of black $\mathrm{TiO}_{2}$ may provide an explanation for the enhanced hydrogen mobility in black $\mathrm{TiO}_{2}$.

To better understand the experimental measurement results of the hydrogenated black $\mathrm{TiO}_{2}$, we preformed $a b$ initio DFT calculations on large $\mathrm{TiO}_{2}$ clusters. As shown in Fig. 5a, model A of $\mathrm{Ti}_{210} \mathrm{O}_{420} \mathrm{H}_{12}$ represents a normal white $\mathrm{TiO}_{2}$ nanocrystal of $\sim 2.5 \mathrm{~nm}$ diameter where the dangling bonds of $\mathrm{O}$ atoms on the surface have been terminated by the $\mathrm{H}$ to form $\mathrm{OH}$ bonds. In model $\boldsymbol{B}, \mathrm{Ti}_{218} \mathrm{O}_{436} \mathrm{H}_{70}$, additional $\mathrm{H}$ atoms have been introduced to simulate the hydrogenation process. After annealing and relaxation, the core of model B remains anatase-like, but its outer shell becomes disordered containing many distorted structures, and the $\mathrm{H}$ atoms are partially bonded to $\mathrm{O}$ and $\mathrm{Ti}$ atoms, as shown as Fig. $\mathbf{5 b}$.

We plotted the calculated electronic density of states (DOS) of models A and B in Fig. 5c. Considering the fact that DFT underestimate the bandgap values, a scissor operator of $1.06 \mathrm{eV}$ has been added to the conduction bands, by which the calculated bandgap of model $\mathbf{a}$ is calibrated to the experimental value of $3.30 \mathrm{eV}$, and the calculated energy of mid-gap states have been linearly enlarged by a
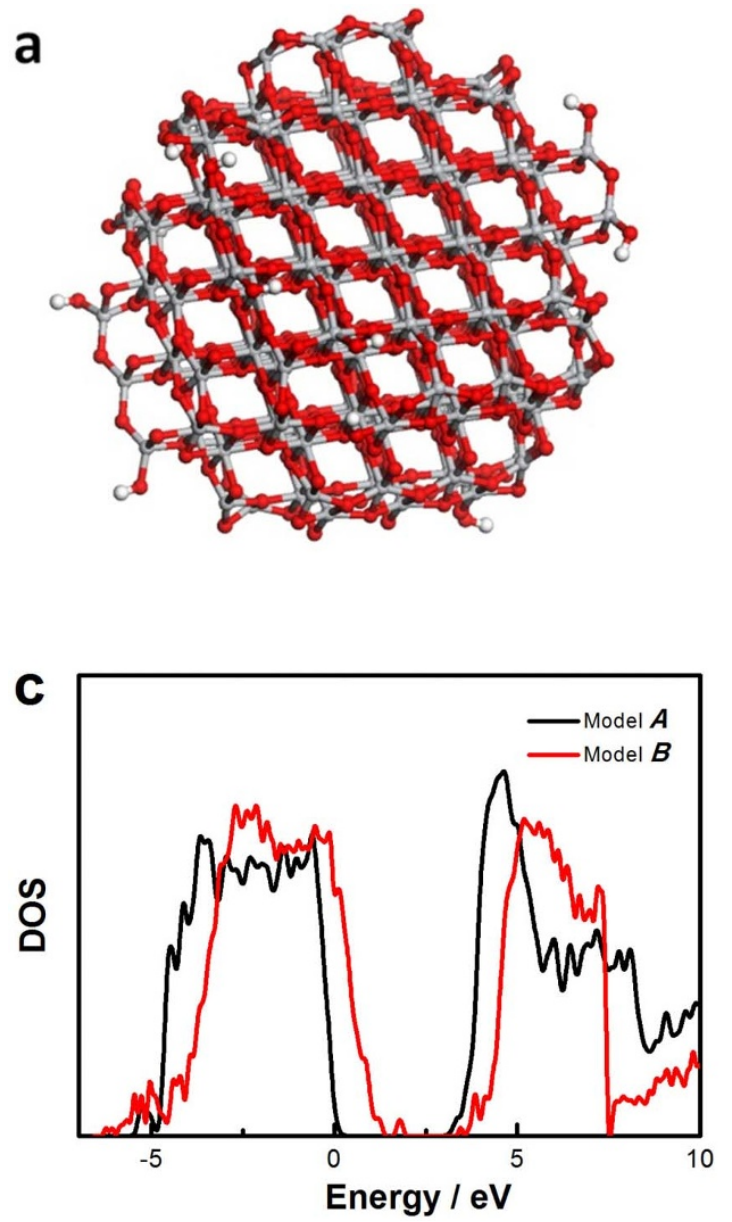

scaling parameter of 1.48 accordingly. Clearly model $B$ results in the appearance of new states which extend from the valence band to more than $1.20 \mathrm{eV}$ above the valence band maximum (VBM). There is also evidence of a small number of mid-gap states centered at about $1.80 \mathrm{eV}$ above the VBM. The blue-shift of the VBM and reduction of the band gap agrees with the experimental XPS results and optical absorption in black $\mathrm{TiO}_{2}$. The DOS of model B shows a blueshift of the VB M by $\sim 1.8 \mathrm{eV}$. In comparison with the $2.18 \mathrm{eV}$ upshift of the VBM observed experimentally ${ }^{10}$, there is a difference of about $0.38 \mathrm{eV}$. While having much bigger size than model $\mathrm{B}$, the real black $\mathrm{TiO}_{2}$ nano-clusters may contain more lattice distortions and with more mid-gap states to broaden their electronic DOS. From the vibrational DOS of $\mathrm{H}$ atoms in model $\boldsymbol{B}$ (Fig. $5 \mathrm{~d}$ ), the dynamics of hydrogen in black $\mathrm{TiO}_{2}$ can be divided into four frequency regions. The highest frequency modes around $4200 \mathrm{~cm}^{-1}$ are due to the stretching modes of the $\mathrm{H}-\mathrm{H}$ bonds. That means some hydrogen atoms are trapped somewhere in the nanocrystal core or the disordered layer in the form of $\mathrm{H}_{2}$ molecules, but their amount is small $^{10,27-30}$. These $\mathrm{H}-\mathrm{H}$ stretching modes may not necessarily be infra-red active unless the local symmetry of the $\mathrm{H}_{2}$ molecules is not centro-symmetric. The modes at $\sim 3000 \mathrm{~cm}^{-1}$ and $\sim 1500 \mathrm{~cm}^{-1}$ come from the $\mathrm{OH}$ stretching and bending modes, respectively. Finally, the low frequency modes at around $500 \mathrm{~cm}^{-1}$ and below are produced by hydrogen atoms that are bound weakly to the disordered $\mathrm{TiO}_{2}$ lattice within the surface of model $\boldsymbol{B}$. Unfortunately, these modes overlap with the phonon modes of $\mathrm{TiO}_{2}$ and as a result they may not be observed easily in the IR spectra. Since the hydrogen atoms are weakly bound they behave like free
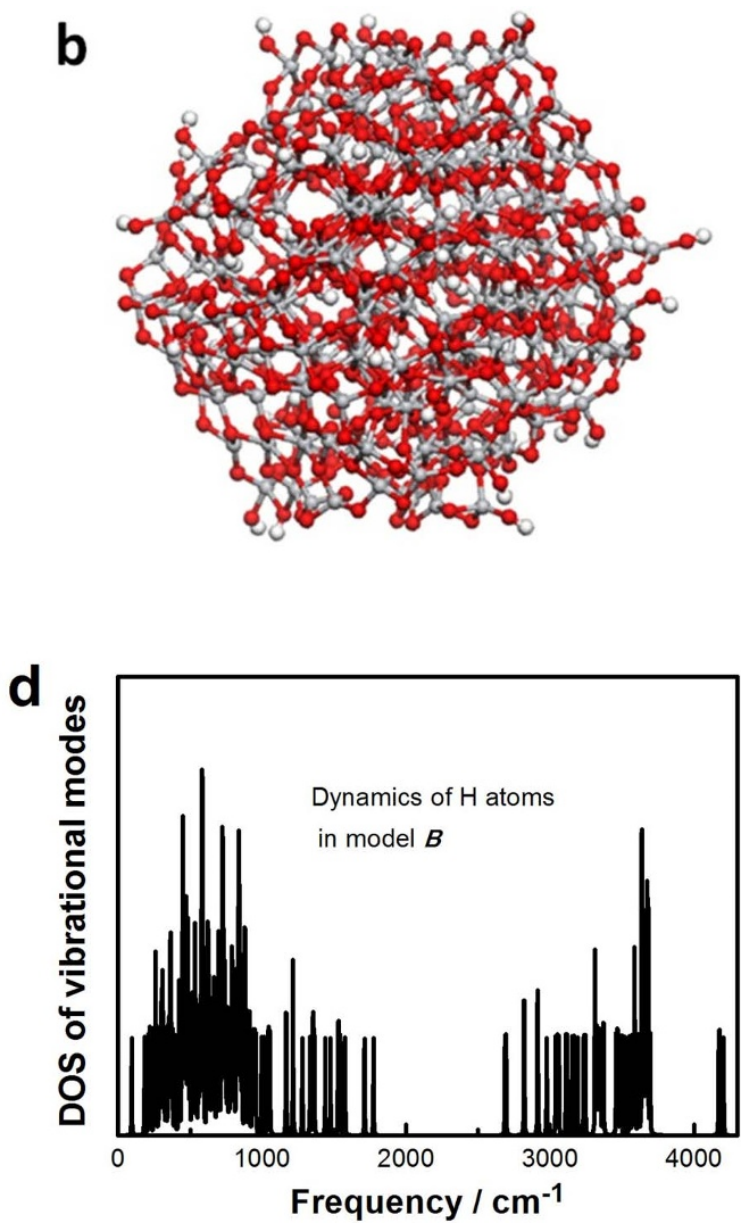

Figure 5 ab initio DFT calculation results on large $\mathrm{TiO}_{2}$ clusters. (a) model A structure of crystalline $\mathrm{Ti}_{210} \mathrm{O}_{420} \mathrm{H}_{12}$. (b) model B structure of disordered $\mathrm{Ti}_{218} \mathrm{O}_{436} \mathrm{H}_{70}$, Ti, $\mathrm{O}$ and $\mathrm{H}$ atoms are shown as grey, red and white balls, respectively. (c) The calculated electronic DOS of models A and B. The zero of the energy scale is set at the top of the valence band. (d) The calculated vibrational DOS of model B. 
hydrogen and can diffuse readily from one site to another. This may account for the new sharp NMR peaks which appear in black $\mathrm{TiO}_{2}$ and have chemical shifts near zero ppm.

In summary, the rich physics of hydrogenated black $\mathrm{TiO}_{2}$ nanoparticles distinguish themselves from all known $\mathrm{TiO}_{2}$-based nanomaterials, white or coloured. Black $\mathrm{TiO}_{2}$ absorbs in the visible and infrared wavelength region, but unlike in traditional doped $\mathrm{TiO}_{2}$, $\mathrm{Ti}^{3+}$ defects or impurities are not responsible for the long-wavelength absorption of black $\mathrm{TiO}_{2}$. Hydrogen induces a disordered phase on the outside of crystalline anatase nanoparticle core. The hydrogen can move rapidly around, and yield electronic structure changes from the alteration of the orbital overlapping in $\mathrm{TiO}_{2}$. These unique signatures would enable black $\mathrm{TiO}_{2}$ nanoparticles to improve the performance and efficiency of a range of solar energy systems, as already seen in a significantly enhanced solar-driven photocatalytic activity.

\section{Methods}

White $\mathrm{TiO}_{2}$ nanocrystals were first prepared with a precursor solution consisting of titanium tetraisopropoxide, ethanol, hydrochloric acid, deionized water, and an organic template, Pluronic F127. The solution was heated at $40^{\circ} \mathrm{C}$ for 24 hours and then dried at $110^{\circ} \mathrm{C}$ for 24 hours. The dried powders were calcinated at $500^{\circ} \mathrm{C}$ for 6 hours to remove the organic template and enhance the crystallization of $\mathrm{TiO}_{2}$. The resulting white powders were first maintained in a vacuum for 1 hour and then hydrogenated in a 20.0 -bar $\mathrm{H}_{2}$ atmosphere at about $200^{\circ} \mathrm{C}$ for 5 days to get black $\mathrm{TiO}_{2}$ nanocrystals. The UV-vis-NIR spectra were measured on a Cary $5000 \mathrm{UV}$ vis-NIR spectrometer. About $50 \mathrm{mg}$ of $\mathrm{TiO}_{2}$ nanocrystals were pressed into the small sample container to make a flat surface and uniform density in a praying mantis diffuse reflection accessory. The FTIR spectra were measured on a Nicolet 6700 FT-IR Spectrometer with an attenuated total reflectance (ATR) unit. Small amount of the samples were pressed onto the ATR window for the measurements. All the measurements were conducted under ambient conditions in air. The baseline was corrected against air under the sample measurement parameters. The HRTEM images were taken on a Phillips CM 200 microscope. A small amount of sample was first dispersed in water by sonication. One drop of the aqueous suspension was then transferred onto a thin holey carbon film. The grids were then dried in an oven at $60^{\circ} \mathrm{C}$ overnight before TEM examination. The samples did not change during these preparations. The X-ray spectroscopic experiments were performed at the undulator beamline 7.0.1 of the Advanced Light Source (ALS), Lawrence Berkeley National Laboratory (LBNL), with a spherical grating monochromator ${ }^{31}$. Ti $2 p\left(L_{2}, 3\right)$ XES and $\mathrm{O} 1 s(K \alpha)$ XES spectra were recorded by using a Nordgren-type grating spectrometer. The spectrometer was mounted perpendicular to the incoming photon beam in the polarization plane and the resolution was $0.3 \mathrm{eV}$ and $0.4 \mathrm{eV}$, respectively, for $\mathrm{Ti}$ $L$ - and $\mathrm{O} K$-edge XES spectra. The monochromator was set to the excitation energies of $475 \mathrm{eV}$ and $565 \mathrm{eV}$ respectively, for $\mathrm{Ti} L$ and $\mathrm{O} K$ spectra with a resolution of $0.5 \mathrm{eV}$. The samples were mounted to have a beam incidence angle of $30^{\circ}$ to the sample surface. For energy calibration of the Ti $L$ - and $\mathrm{O} K$-XES, the spectra of the reference samples $\mathrm{Ti}$ and $\mathrm{TiO}_{2}$ were measured. The base pressure of the chamber was $2 \times 10^{-9}$ Torr. The absorption spectra at the Ti $2 p$ and $\mathrm{O} 1 s$ edges were measured by means of total electron yield (TEY) with a monochromator resolution set to $0.2 \mathrm{eV}$. The absorption intensity was normalized by the current from a clean gold mesh placed in the incoming beam to compensate for fluctuations of the incoming photon intensity. The XES and XAS spectra were brought to a common energy scale using an elastic peak in the emission spectra recorded at the excitation energy set at the absorption edge. The measurement depth of XAS as measured by TEY at these edges is normally around $10 \mathrm{~nm}$, and is around $100 \mathrm{~nm}$ for XES. Ti K-edge X-ray absorption measurements were performed in fluorescence mode at beamline 10.3.2 at the $\mathrm{ALS}^{32}$. Samples were spread on tape, and spots with relatively low count rates were chosen in order to avoid the over-absorption ("self-absorption") effect. The probe depth for this method is about $5 \mu \mathrm{m}$, assuming the bulk anatase density. Spectra taken on different spots were consistent with each other. The edge for Ti foil was taken to be $4966.40 \mathrm{eV}$. The QuickXAS mode was used for data collection. Data were processed using standard software available at the beamline and from its website ${ }^{33}$. No radiation-induced effects were seen by comparing successive scans. We also performed high-resolution XPS and XAS experiments at the bending-magnet beamline 9.3.2 at ALS, $\mathrm{LBNL}^{34}$. The endstation can perform photoemission experiments up to a pressure of 1 torr. Incident photon energy can be tuned to adjust the kinetic energy of photoelectrons from the Ti $2 p$, and $\mathrm{O} 1 s$ core levels. The probing depth for the photon energies used in this study is about $1 \mathrm{~nm}$. The energy resolution of the beam line is 3000. All NMR experiments were performed on an 8.45 tesla Oxford magnet with a three channel Tecmag Apollo NMR console, using a home-built, doubly tuned, MAS solid-state NMR probe with a $3.2 \mathrm{~mm}$ stator assembly from Revolution NMR. The magic angle spinning (MAS) rate was $12 \mathrm{kHz}$, the proton excitation pulse during the Bloch decay experiment was $\sim 50 \mathrm{kHz}$, and the spectra were externally referenced to adamantane. Each spectrum was acquired with 16 scans and the subtraction was performed after normalizing the peak at $\sim 6 \mathrm{ppm}$. Both white and black $\mathrm{TiO}_{2}$ samples were evacuated at room temperature overnight to remove the surface adsorbed water vapor from the atmosphere.
All the theoretical calculations were based on the first-principles density-functional theory (DFT) using the Perdew-Burke-Ernzerhof (PBE) generalized gradient approximation, with the Kohn-Sham equations solved with the projected augmented wave method as implemented in the VASP code ${ }^{35,36}$. All models were based on $30 \times 30 \times 30 \AA^{3}$ supercells, where the atomic positions are relaxed until their residual forces are less than $0.05 \mathrm{eV}^{-1}$. The cut-off energy for the plane-wave basis set was $400 \mathrm{eV}$ and the Brillouin zone was sampled with the single $\Gamma$-point.

1. Fujishima, A. \& Honda, K. Electrochemical photolysis of water at a semiconductor electrode. Nature 238, 37-38 (1972).

2. Grätzel, M. Photoelectrochemical cells. Nature 414, 338-344 (2001).

3. Chen, X. \& Burda, C. The electronic origin of the visible-light absorption properties of C-, $\mathrm{N}$ - and S-doped $\mathrm{TiO}_{2}$ nanomaterials. J. Am. Chem. Soc. 130, 5018-5019 (2008).

4. Chen, X. \& Mao, S. S. Titanium dioxide nanomaterials: Synthesis, properties, modifications, and applications. Chem. Rev. 107, 2891-2959 (2007).

5. Khan, S. U. M., Al-Shahry, M. \& Ingler, W. B., Jr. Efficient photochemical water splitting by a chemically modified $\mathrm{n}-\mathrm{TiO}_{2}$. Science 297, 2243-2245 (2002).

6. Asahi, R., Morikawa, T., Ohwaki, T., Aoki, K. \& Taga, Y. Visible-light photocatalysis in nitrogen-doped titanium oxides. Science 293, 269-271(2001).

7. Choi, W., Termin, A. \& Hoffmann, M. R. The role of metal ion dopants in quantum-sized $\mathrm{TiO}_{2}$ : correlation between photoreactivity and charge carrier recombination dynamics. J. Phys. Chem. 98, 13669-13679 (1994).

8. Anpo, M. \& Takeuchi, M. The design and development of highly reactive titanium oxide photocatalysts operating under visible light irradiation. J. Catal. 216, 505516 (2003).

9. Zuo, F. et al. Self-doped $\mathrm{Ti}^{3+}$ enhanced photocatalyst for hydrogen production under visible light. J. Am. Chem. Soc. 132, 11856-11857 (2010).

10. Chen, X., Liu, L., Yu, P. Y. \& Mao, S. S. Increasing solar absorption for photocatalysis with black hydrogenated titanium dioxide nanocrystals. Science 331, 746-750 (2011).

11. Naldoni, A. et al. Effect of nature and location of defects on bandgap narrowing in black $\mathrm{TiO}_{2}$ nanoparticles. J. Am. Chem. Soc. 134, 7600-7603 (2012).

12. Pan, H., Zhang, Y.-W., Shenoy, V. B. \& Gao, H. Effects of H-, N-, and (H, N)doping on the photocatalytic activity of $\mathrm{TiO}_{2}$. J. Phys. Chem. C 115, 12224-12231 (2011).

13. Syzgantseva, O. A., Gonzalez-Navarrete, P., Calatayud, M., Bromley, S. \& Minot, C. Theoretical Investigation of the Hydrogenation of $\left(\mathrm{TiO}_{2}\right)_{\mathrm{N}}$ Clusters (N = 1-10). J. Phys. Chem. C 115, 15890-15899 (2011).

14. Wang, G. et al. Hydrogen-treated $\mathrm{TiO}_{2}$ nanowire arrays for photoelectrochemical water splitting. Nano Lett. 11, 3026-3033 (2011).

15. Diebold, U. The surface science of titanium dioxide. Surf. Sci. Rep. 48, 53-229 (2003).

16. Sun, C. et al. Hydrogen incorporation and storage in well-defined nanocrystals of anatase titanium dioxide. J. Phys. Chem. C 115, 25590-25594 (2011).

17. Kunat, M., Burghaus, U. \& Wöll, C. The adsorption of hydrogen on the rutile $\mathrm{TiO}_{2}$ (110) surface. Phys. Chem. Chem. Phys. 6, 4203-4207 (2004).

18. Suzuki, S., Fukui, K. I., Onishi, H. \& Iwasawa, Y. Hydrogen adatoms on $\mathrm{TiO}_{2}(110)-(1 \times 1)$ characterized by scanning tunneling microscopy and electron stimulated desorption. Phys. Rev. Lett. 84, 2156-2159 (2000).

19. Fujino, T., Katayama, M., Inudzuka, K., Okuno, T. \& Oura, K. Surface hydroxyl formation on vacuum-annealed $\mathrm{TiO}_{2}$ (110). Appl. Phys. Lett. 79, 2716-2718 (2001).

20. Yin, X.-L. et al. Diffusion versus desorption: complex behavior of $\mathrm{H}$ Atoms on an oxide surface. ChemPhysChem 9, 253-256 (2008).

21. Di Valentin, C., Pacchioni, G. \& Selloni, A. Reduced and n-type doped $\mathrm{TiO}_{2}$ : nature of $\mathrm{Ti}^{3+}$ species. J. Phys. Chem. C 113, 20543-20552 (2009).

22. Islam, M. M., Calatayud, M. \& Pacchioni, G. Hydrogen adsorption and diffusion on the anatase $\mathrm{TiO}_{2}(101)$ surface: a first-principles investigation. J. Phys. Chem. C 115, 6809-6814 (2011).

23. de Groot, F. M. F. et al. Oxygen 1s x-ray absorption of tetravalent titanium oxides: A comparison with single-particle calculations. Phys. Rev. B 48, 2074-2080 (1993).

24. Cracker, M. et al. ${ }^{1} \mathrm{H}$ NMR spectroscopy of titania - chemical shift assignments for hydroxy groups in crystalline and amorphous forms of $\mathrm{TiO}_{2}$. J. Chem. Soc. Faraday Trans. 92, 2791-2798 (1996).

25. Jonsen, P. Identification of different hydrogen-reduced titania crystallographis forms by ${ }^{1} \mathrm{H}$ NMR spectroscopy. Catal. Lett. 2, 345-350 (1989).

26. Jonsen, P. A. ${ }^{1} \mathrm{H}$ NMR Study of Reduced $\mathrm{Ru} / \mathrm{TiO}_{2}$ and $\mathrm{TiO}_{2}$. Colloids Surf. 36, 127-132 (1989)

27. Bavykin, D. V., Lapkin, A. A., Plucinski, P. K., Friedrich, J. M. \& Walsh, F. C. Reversible storage of molecular hydrogen by sorption into multilayered $\mathrm{TiO}_{2}$ nanotubes. J. Phys. Chem. C 109, 19422-19427 (2005).

28. Lim, S. H., Luo, J., Zhong, Z., Ji, W. \& Lin, J. Room-temperature hydrogen uptake by $\mathrm{TiO}_{2}$ nanotubes. Inorg. Chem. 44, 4124-4126 (2005).

29. Hu, X., Skadtchenko, B. O., Trudeau, M. \& Antonelli, D. M. Hydrogen storage in chemically reducible mesoporous and microporous Ti oxides. J. Am. Chem. Soc. 128, 11740-11741 (2006).

30. Barnard, A. S. \& Curtiss, L. A. Prediction of $\mathrm{TiO}_{2}$ nanoparticle phase and shape transitions controlled by surface chemistry. Nano Lett. 5, 1261-1266 (2005). 
31. Nordgren, J. \& Guo, J.-H. Instrumentation for soft X-ray emission spectroscopy. J. Electr. Spectrosc. Relat. Phenom. 110-111, 1-13 (2000).

32. Marcus, M. A. et al. Beamline 10.3.2 at ALS: a hard X-ray microprobe for environmental and materials sciences. J. Synchrotron Radiation 11, 239-247 (2004).

33. Ravel, B. \& Newville, M. ATHENA, ARTEMIS, HEPHAESTUS: data analysis for $X$-ray absorption spectroscopy using IFEFFIT. J. Synchrotron Rad. 12, 537-541 (2005).

34. Grass, M. E. et al. New ambient pressure photoemission endstation at Advanced Light Source beamline 9.3.2. Rev. Sci. Instrum 81, 053106 (2010).

35. Kresse, G. \& Furthmüller, J. Efficient iterative schemes for ab initio total-energy calculations using a plane-wave basis set. Phys. Rev. B 54, 11169-11186(1996).

36. Kresse, G. \& Joubert, D. From ultrasoft pseudopotentials to the projector augmented-wave method. Phys. Rev. B 59 1758-1775 (1999).

\section{Acknowledgements}

This research has been supported by the Office of Energy Efficiency and Renewable Energy of the U.S. Department of Energy. TEM work was performed at the National Center for Electron Microscopy, which is supported by the Office of Science, Office of Basic Energy Sciences of the U.S. Department of Energy. The operations of the Advanced Light Source at Lawrence Berkeley National Laboratory are supported by the Director, Office of Science, Office of Basic Energy Sciences, U.S. Department of Energy under contract number DE-AC02-05CH11231. X.C. thanks the support from College of Arts and Sciences,

University of Missouri - Kansas City and University of Missouri Research Board. LL thanks support from the National Natural Science Foundation of China (No. 11174273) and the 100 Talents Program of the Chinese Academy of Sciences.

\section{Author contributions}

X.C. and S.S.M. conceived the experimental research; L.L. and P.Y.Y. constructed the theoretical model. X.C. conducted UV-vis-NIR, FTIR, and HRTEM measurements; L.L. and P.Y.Y. did the calculations; Z.L., M.E.G. and B.M. performed XPS measurements; M.A.M. conducted Ti K-edge XANES experiments; W.-C.W., P.-A.G. and J.G. carried out XAS and XES characterizations; and N.A.O. did NMR testing. X.C. and S.S.M. wrote the manuscript with contribution from all authors; everyone participated in discussions and analysis of the results.

\section{Additional information}

Supplementary information accompanies this paper at http://www.nature.com/ scientificreports

Competing financial interests: The authors declare no competing financial interests.

License: This work is licensed under a Creative Commons

Attribution-NonCommercial-ShareAlike 3.0 Unported License. To view a copy of this license, visit http://creativecommons.org/licenses/by-nc-sa/3.0/

How to cite this article: Chen, X. et al. Properties of Disorder-Engineered Black Titanium Dioxide Nanoparticles through Hydrogenation. Sci. Rep. 3, 1510; DOI:10.1038/srep01510 (2013). 